6-1-2018

\title{
Improving Pharmacologic Prevention of VTE in Trauma: IMPACT- IT QI Project
}

\author{
Audis Bethea \\ Charleston Area Medical Center \\ Elliot Adams \\ Charleston Area Medical Center \\ Frank C. Lucente \\ West Virginia University \\ Damayanti Samanta \\ Charleston Area Medical Center \\ Julton Tomanguillo Chumbe \\ Charleston Area Medical Center
}

Follow this and additional works at: https://researchrepository.wvu.edu/ctsi

Part of the Medicine and Health Sciences Commons

\section{Digital Commons Citation}

Bethea, Audis; Adams, Elliot; Lucente, Frank C.; Samanta, Damayanti; and Chumbe, Julton Tomanguillo, "Improving Pharmacologic Prevention of VTE in Trauma: IMPACT-IT QI Project" (2018). Clinical and Translational Science Institute. 960.

https://researchrepository.wvu.edu/ctsi/960

This Article is brought to you for free and open access by the Centers at The Research Repository @ WVU. It has been accepted for inclusion in Clinical and Translational Science Institute by an authorized administrator of The Research Repository @ WVU. For more information, please contact ian.harmon@mail.wvu.edu. 


\title{
Improving Pharmacologic Prevention of VTE in Trauma: IMPACT- IT QI Project
}

\author{
AUDIS BETHEA, PharmD, B.C.P.S. ${ }^{*}$ ELLIOT ADAMS, M.D. ${ }^{\dagger}$, FRANK C. LUCENTE, M.D. ${ }^{\ddagger}$ \\ DAMAYANTI SAMANTA, M.S. ${ }^{*}$, and JULTON TOMANGUILLO CHUMBE, M.D. \\ "Center for Health Services and Outcomes Research, Charleston Area Medical Center, \\ Charleston, West Virginia; \\ †Charleston Area Medical Center, Charleston, West Virginia; \\ $\mp$ West Virginia University Physicians of Charleston, Charleston Area Medical Center, Charleston, \\ West Virginia
}

\section{Abstract}

Enoxaparin regimens commonly used for prophylaxis fail to achieve optimal anti-factor Xa levels in up to 70 per cent of trauma patients. Accordingly, trauma services at the study institution endeavored to develop a standardized approach to optimize pharmacologic prevention with enoxaparin. An enoxaparin venous thromboembolism (VTE) prophylaxis protocol implemented in October 2015 provided weight-adjusted initial dosing parameters with subsequent dose titration to achieve targeted anti-factor Xa levels. Symptomatic VTE rate was evaluated 12 months pre- and post-implementation. Data were obtained from the trauma registry and charts were reviewed from electronic medical records. The rate of symptomatic VTE significantly declined postimplementation (2.0\% vs $0.9 \%, P=0.009)$. Enoxaparin use was comparable in these two phases validating that the decline in symptomatic VTEs was not due to an increase in enoxaparin use. Symptomatic VTE rate for patients who received enoxaparin in the post-implementation cohort decreased from 3.2 to 1.0 per cent $(P=0.023,95 \%$ confidence interval $=0.124-0.856)$. There was also a significant decrease in the rate of symptomatic deep vein thrombosis $(2.8 \%$ vs $0.9 \%, P=$ $0.040,95 \%$ confidence interval $=0.117-0.950$ ). This approach to VTE prophylaxis with enoxaparin resulted in a significant reduction in symptomatic VTE rates. Implementation of similar practices may be equally impactful in other institutions that use enoxaparin.

Trauma patients carry an especially high risk for venous thromboembolism (VTE). ${ }^{1,2}$ The incidence of deep vein thrombosis (DVT) after severe trauma has been estimated to be as high as 70 per cent in patients not receiving preventative therapies. ${ }^{1,2}$ Sequelae of DVTs, such as pulmonary embolism (PE), have been reported as the third leading cause of death in trauma patients surviving past the initial 24 hours. ${ }^{3,4}$ Numerous factors contribute to the frequency of VTE development after severe trauma, including injury type and severity,

Reproduced with permission of copyright owner. Further reproduction prohibited without permission.

Address correspondence and reprint requests to Audis Bethea, PharmD, B.C.P.S., Clinical Research Scientist/Pharmacy Specialist, Trauma/Surgery Research Scientist, West Virginia Clinical and Translational Science Institute, 3211 MacCorkle Avenue SE, Charleston, WV 25304. audis.bethea@camc.org. 
requirements for massive transfusion, surgical intervention, immobility, extended hospital stays, and acute inflammatory processes. ${ }^{1-9}$ Accordingly, present guidelines recommend that all trauma patients without contraindications receive VTE prophylaxis. ${ }^{1,4}$

Benchmarking data from the American College of Surgeons Trauma Quality Improvement Program (TQIP) suggest that 45 to 60 per cent of all trauma patients admitted to United States hospitals receive pharmacologic VTE prophylaxis. ${ }^{10}$ Recommended agents for pharmacologic prophylaxis in this patient population are low-molecular weight heparins (LMWH). ${ }^{8,9,11}$ These recommendations are reflected in TQIP data suggesting that 70 per cent of the patients who receive pharmacologic VTE prophylaxis are administered LMWHs. Although these agents are the most frequently used modality for pharmacologic prevention, only 35 to 40 per cent of all trauma admissions receive pharmacologic prophylaxis with LMWH. Despite the relatively low rate of pharmacologic prophylaxis with LMWH, TQIP cites an average DVT and PE rate of 1.5 per cent and 0.6 per cent, respectively. ${ }^{10}$

At the study institution, enoxaparin is the most widely used LMWH. The recommended enoxaparin dosing regimen for prophylaxis is $30 \mathrm{mg}$ twice daily administered subcutaneously (SQ). ${ }^{8,9,11}$ Although enoxaparin does not require laboratory monitoring, the efficacy can be monitored by measuring anti-factor Xa levels. A peak anti-factor Xa level of 0.2 to $0.5 \mathrm{IU} / \mathrm{mL}$ has previously been determined to predict adequate VTE prophylaxis. 12-14 However, there is recent evidence that standard enoxaparin dosing may be insufficient to achieve prophylactic anti-factor Xa levels in up to 70 per cent of all trauma patients. ${ }^{2,15}$

Venous thromboembolism rates at the present study's institution exceeded TQIP benchmarked averages for 2015. Accordingly, the trauma service at the study institution endeavored to improve its performance with respect to VTE prevention. With a growing body of evidence suggesting suboptimal efficacy with routinely used enoxaparin regimens, the approach to the provision of pharmacologic prophylaxis was amended. A protocol consisting of an initial, weight-adjusted enoxaparin regimen with subsequent dosing adjustments guided by anti-factor Xa levels was developed. The aim of this quality improvement analysis was to use this targeted approach to pharmacologic prophylaxis to reduce the rate of symptomatic VTEs.

\section{Materials and Methods}

\section{Protocol Development}

Historically, pharmacologic VTE prophylaxis in the trauma patient population was not standardized at the study institution. Trauma services VTE Prophylaxis Algorithm prompted the implementation of pharmacologic prophylaxis but did not specify dosing strategies or pharmacologic modalities. The enoxaparin VTE prophylaxis protocol added pharmacologic management components to trauma services' VTE Prophylaxis Algorithm (Fig. 1). The updated algorithm and enoxaparin protocol was approved in October 2015 as a quality improvement endeavor focused on improving trauma patient outcomes through guided pharmacologic prevention of VTEs. Patients without contraindication(s) received an initial dose of enoxaparin based on their weight. Enoxaparin $30 \mathrm{mg}$ administered SQ every 12 hours was ordered if weight was $<100 \mathrm{~kg}$ and $40 \mathrm{mg}$ SQ every 12 hours when weight was 
$\geq 100 \mathrm{~kg}$. Dose administrations were scheduled for noon and midnight. Anti-factor Xa levels were checked with morning labs (approximately 4 AM) after the midnight administration of the $3 \mathrm{rd}$ or 4 th dose. If a subtherapeutic anti-factor Xa level was reported $(<0.2 \mathrm{IU} / \mathrm{mL})$, each scheduled dose of enoxaparin was increased by 20 per cent. If a super-therapeutic level of anti-factor Xa level was reported $(>0.5 \mathrm{IU} / \mathrm{mL})$, each scheduled dose of enoxaparin was decreased by 20 per cent. Adjusted doses were rounded to the nearest zero. After each dosing change, anti-factor Xa levels were reordered after the same process and adjusted accordingly. This process was followed until a therapeutic anti-factor Xa level for prophylaxis $(0.2-0.5 \mathrm{IU} / \mathrm{mL})$ was achieved. For a patient whose initial $30 \mathrm{mg}$ SQ every 12 hours or $40 \mathrm{mg}$ SQ every 12 hours regimen resulted in a supertherapeutic anti-factor Xa level, the dosing frequency was reduced to once daily and no additional anti-factor Xa levels were evaluated.

Trauma services enoxaparin VTE prophylaxis protocol was contraindicated in patients with renal insufficiency (GFR/CrCL $<30 \mathrm{~mL} / \mathrm{min}$ ), thrombocytopenia $<50,000 / \mathrm{mcL}$, or the presence of injuries or clinical conditions outlined in the service's VTE Prophylaxis Algorithm (Fig. 1). Anti-factor Xa levels for monitoring enoxaparin were designated as "Lovenox Heparin Assays" by laboratory services at the study institution and are accordingly referenced as such in the protocol presented in Figure 2.

\section{Patients and Study Design}

Patients admitted to trauma services between October 2014 and September 2016 were included in the study. Symptomatic VTE rate was evaluated 12 months before (October 2014 to September 2015) and after (October 2015 to September 2016) implementation of the Trauma Services Enoxaparin VTE Prophylaxis protocol. Data were obtained from the trauma registry and patient charts were reviewed from electronic medical records. General demographics, Injury Severity Score (ISS), pre-existing conditions, presenting injuries, incidence of intensive care unit (ICU) admission, ICU and hospital length of stay (LOS), rate of massive transfusion, surgical procedures, and diagnosis of symptomatic VTE in the post-implementation phase were prospectively collected by the trauma registrars. Additional data extraction, including patients' body mass index (BMI), enoxaparin dosing, anti-factor Xa level(s), date and time of first enoxaparin administration, frequency of enoxaparin use, and enoxaparin failure rates were manually collected. For the purpose of this study, enoxaparin failure rate was operationalized as the development of a VTE after the administration of at least one prophylactic dose of enoxaparin. Venous thromboembolisms documented by the trauma registry were confirmed by investigators to be in compliance with the "ACS NTDB National Trauma Data Standard: Data Dictionary for 2016 Admissions." 15

Surveillance studies for VTE in patients receiving pharmacologic prophylaxis are not regularly performed at the study institution and accordingly were not routinely obtained in this study. Formal studies evaluating patients for the presence of a VTE were ordered and performed when patients' clinical signs and symptomatology warranted a formal workup. Venous duplex ultrasonography and CT arteriography (CTA) was used for the diagnosis of DVT and PE, respectively. 
Descriptive analysis was conducted for each variable. Means and standard deviations were computed for continuous variables and compared using $t$-tests. Data that were not normally distributed were reported as a median with corresponding interquartile range. Categorical variables were presented as proportions and compared using chi-square or Fisher's exact test. Differences in VTE rates for patients who received prophylaxis with enoxaparin were computed with binary logistic regression controlling for variables that significantly differed between the pre- and post-implementation phases. $P$ values of $\$ 0.05$ were considered significant. Analyses were performed using SPSS Version 22.0 (IBM Corp, Armonk, NY). Collection and presentation of this data (study \#16-201) was approved by the CAMC/WVUCharleston Division Institutional Review Board for the Protection of Human Subjects at Charleston Area Medical Center Institute.

\section{Results}

In the 12 months pre- and post-implementation, a total of 1542 and 1802 patients were admitted to trauma services, respectively. The overall rate of symptomatic VTE significantly decreased from pre- to post-implementation $(2.0 \%$ vs $0.9 \%, P=0.009)$. To verify that the significant decline in rate was not the result of increased enoxaparin use, an evaluation of the frequency of enoxaparin prophylaxis in the study groups was conducted. Data revealed that enoxaparin use was comparable in both phases with an overall rate of 30.4 per cent preimplementation and 31.9 per cent post-implementation $(P=0.602)$ (Table 1$)$.

An analysis of patients who received prophylaxis with enoxaparin during the pre- and postimplementation phases was then performed. Patients in both phases were comparable in age, gender, ISS, BMI, and time to first dosing of enoxaparin. Pre-existing conditions were comparable; however, the incidence of pulmonary comorbidities, which were all chronic obstructive pulmonary disease, significantly increased from pre- to post-implementation (2.1\% vs $4.5 \%, P=0.035)$ (Table 2). The incidence of massive transfusions and presenting injuries were also comparable in both time periods (Table 3). An additional comparison of surgical procedures revealed no significant differences in the frequency of interventions between the study cohorts (Table 4).

After adjusting for significant differences in comorbidities, the rate of symptomatic VTEs significantly decreased $(3.2 \%$ vs $1.0 \%, p=0.018,95 \%$ confidence interval $[\mathrm{CI}]=0.121-$ $0.820)$ when the pre- and post-implementation cohorts were compared. Similar trends were noticed when the symptomatic DVT rate was exclusively compared between the pre- and post-implementation groups $(2.8-0.9 \%, P=0.030,95 \% \mathrm{CI}=0.112-0.894)$. There was also a decrease in the post-implementation PE rate; however, this decrease was not significant ( $1.1 \%$ vs $0.2 \%, P=0.246$ ) (Table 5). The duration of hospitalization before VTE diagnosis was compared between the study cohorts. The median time to VTE diagnosis was10.0 [5.522.6] days in the pre-implementation group and 9.72 [3.5-13.7] days in the postimplementation group $(P=0.470)$. In addition, bleeding events were evaluated in the postimplementation cohort. There were four bleeding events that occurred within 48 hours of enoxaparin exposure in the post-implementation phase. All four cases involved erosive or ulcerative gastrointestinal lesions that were subsequently identified via endoscopic or colonoscopic studies. 
Additional variables were evaluated to determine their effect on the incidence of symptomatic VTE between the compared cohorts. Time to initiation of enoxaparin prophylaxis was evaluated. There was no difference in the time to enoxaparin initiation with 2.50 days having elapsed in the pre-implementation group and 2.78 days in the postimplementation group $(P=0.148)$. Initiation of enoxaparin therapy was in compliance with the Enoxaparin Prophylaxis Protocol in 73.8 per cent of patients who received enoxaparin in the post-implementation phase. Average levels were lower in patients who developed symptomatic VTEs versus those who did not; however, the difference did not reach statistical significance $(0.23$ vs $0.34, P=0.061)$. Anti-Xa levels that were not obtained were due to patients' hospitalization being of insufficient duration to allow for assay procurement or protocol noncompliance.

Surveillance studies for VTEs were not routinely performed in the pre- or postimplementation phases. Formal evaluations for VTE via CTA or venous duplex ultrasonography were ordered at the discretion of the trauma service based on clinical suspicion. Accordingly, rates of diagnostic tests for VTEs were evaluated to verify that practices governing the use of these tests did not change between the pre- and postimplementation phases. There were no significant differences in the rate of CTAs performed in the pre- $(5.5 \%)$ versus post-implementation $(5.2 \%)$ phases $(P=0.817)$. Similar rates of duplex ultrasonography studies were also performed between the study groups $(25.7 \% \mathrm{VS}$ $21.5 \%, P=0.109$ ) (Table 6).

\section{Discussion}

The heightened risk of VTE in trauma patients has been consistently documented throughout the literature. ${ }^{1-9,11,15}$ As previously mentioned, the hyper-coagulable state experienced by patients after severe trauma is thought to be multifactorial. Several risk factors are commonplace in the hospitalized patient and are not unique to those who experience trauma. A few risk factors, however, such as massive transfusion, orthopedic injury, and the need for frequent surgical intervention are fairly unique. Of note, it is estimated that approximately 25 per cent of all patients with severe trauma present with a coagulopathy. ${ }^{16-22}$ Those presenting with severe coagulopathy will often require massive transfusion. Coagulopathic patients surviving the initial 24 hours may develop a subsequent, prolonged hypercoagulable state that is thought to be influenced by the early depletion of endogenous protein C. ${ }^{16-22}$ This physiologic process likely has an additive effect in predisposing these patients to hypercoagulability. In addition, the hypercoagulable state that ensues after severe polytrauma may persist for up to three weeks after injury(s). ${ }^{7}$ There also seems to be an escalation in hypercoagulability for up to 10 days in a subset of patients who receive surgical intervention for orthopedic injuries. ${ }^{5}$ As a result, VTE continues to be a significant cause of morbidity and mortality in the trauma patient population. Implementation of routine strategies targeted at minimizing the occurrence of VTEs has proven to be effective. This is indicated by the low incidence of VTE in the present study population at baseline (2.0\%). Indeed, the relatively low frequency of VTE found in this study has been supported by previous data where mechanical and/or pharmacologic prevention is practiced. ${ }^{7}$ 
Despite the relatively low incidence of VTE with the implementation of preventative therapies, the impact on morbidity and mortality can be profound. A study of 7937 trauma patients conducted by Paffrath et al. found that the development of VTE after trauma occurred in approximately 1.8 per cent $(n=146)$ of patients. The incidence of sepsis, multiple organ failure, and hospital LOS, however, were all significantly higher for patients who developed a post-trauma VTE. In addition, the adjusted odds ratio for in-hospital mortality was doubled for patients who developed a post-trauma VTE. In the 146 patients of Paffrath's study who developed a VTE, 118 (80.8\%) were receiving either mechanical or pharmacologic prophylaxis at the time of VTE diagnosis. ${ }^{7}$ Despite the low incidence of post-trauma VTE when prophylaxis is used, the sequelae associated with its failure can be significant.

The potential efficacy of prophylaxis with LMWH has been shown to be enhanced when therapy achieves optimal anti-factor Xa levels. ${ }^{2}, 15$ Monitoring of anti-factor Xa levels, however, has traditionally been reserved for patients who are considered to have altered LMWH pharmacokinetics such as obese, geriatric, and renal dysfunction patients. ${ }^{12,} 14$ Recent literature suggests that this paradigm may need to be revisited as derangements in the pharmacokinetics of LMWH may not be limited to these previously highlighted populations. $2,15,23,24$

The presence of altered LMWH pharmacokinetics and resulting suboptimal anti-factor Xa levels has been linked to increased VTE rates in the trauma patient population when traditional enoxaparin regimens of $30 \mathrm{mg}$ SQ every 12 hours have been studied. Malinoski et al. reported the results of a study evaluating the efficacy of $30 \mathrm{mg}$ of enoxaparin administered SQ twice daily in 54 critically ill trauma and surgery patients. In this study, peak anti-Xa levels were drawn after the third dose of enoxaparin. Subtherapeutic levels $(\$ 0.1 \mathrm{IU} / \mathrm{mL})$ were recorded in 50 per cent $(\mathrm{n}=27)$ of the patients. The DVT rate in this subtherapeutic group was significantly higher than that of the patients who achieved therapeutic anti-factor Xa levels ( $37 \%$ vs $11 \%, P=0.026){ }^{2}$ An additional prospective study by Costantini et al. ${ }^{15}$ measured anti-factor Xa levels in 61 trauma patients also receiving 30 $\mathrm{mg}$ of enoxaparin SQ twice daily. In this study, the regimen resulted in subtherapeutic antifactor Xa levels ( $₫) .2 \mathrm{IU} / \mathrm{mL}$ ) in 70 per cent of patients.

These studies suggest that higher doses of enoxaparin may improve the achievement of therapeutic anti-factor Xa levels, simultaneously improving clinical efficacy in the prevention of VTEs. This hypothesis was supported by a study conducted by Nunez et al., which compared the frequency of achieving therapeutic anti-factor Xa levels between historic (30 mg of enoxaparin SQ every 12 hours) and a prospective cohort that received $0.6 \mathrm{mg} / \mathrm{kg}$ SQ every 12 hours of enoxaparin. Therapeutic anti-factor Xa levels were achieved in 8 per cent of the historic cohort and 61 per cent of the weight-based dosing cohort $(P<$ 0.0001 ). The authors of this study concluded that a weight-based enoxaparin dosing strategy of $0.6 \mathrm{mg} / \mathrm{kg}$ administered SQ every 12 hours may provide a superior strategy for VTE prophylaxis in critically ill trauma patients. ${ }^{3}$

The present quality improvement study evaluated the efficacy of a weight-adjusted therapy for the initiation of pharmacologic prevention of symptomatic VTE with further dosing 
adjustments guided by the prospective evaluation of anti-factor Xa assays. With the implementation of this pharmacotherapeutic approach, the trauma service's overall rate of symptomatic VTE significantly decreased. The enoxaparin protocol also facilitated a significant improvement in pharmacologic prevention, as evidenced by the decrease in symptomatic VTEs during the post-implementation phase for patients who received prophylaxis with enoxaparin.

Despite this study's large sample size and the considerable improvement elicited in the frequency of symptomatic VTE and enoxaparin failures, the study is not without limitations. The data for the pre-implementation and a portion of the post-implementation cohorts were collected retrospectively. Accordingly, this data could have been affected by inaccuracies in the medical record and data extraction errors. In addition, noncompliance with the protocol may have been influenced by the frequent turnover of resident staff and the need for continued education concerning the new practice. The incidence of pharmacologic prophylaxis with enoxaparin was just above 30 per cent in both the pre- and postimplementation study groups. Although this seems to be a low rate of pharmacologic prophylaxis, it is consistent with national benchmarking data provided in American College of Surgeons TQIP reports for 2015 and 2016. In these reports, LMWH was used for the provision of pharmacologic VTE prophylaxis for approximately 35 to 36 per cent of all patients. ${ }^{10}$ Finally, given the inherent limitations of a quality improvement study; it was not within the scope of this study to evaluate this approach to enoxaparin prophylaxis on the development of asymptomatic VTEs.

\section{Conclusions}

Given the significant impact of VTEs on morbidity and mortality in trauma patients who experience these post-injury complications, significant efforts should be taken to ensure that preventative therapy is consistently optimized. This study provides clinical evidence supporting the efficacy of an original protocol for the management of enoxaparin in the prevention of symptomatic VTEs in the trauma patient population. This weight-adjusted approach to initial dosing followed by additional titration(s) based on anti-factor Xa levels resulted in a dramatic decrease in symptomatic VTE rates. This approach offers an efficacious management strategy that could be implemented by other institutions that use enoxaparin for prophylaxis and have the ability to evaluate enoxaparin therapy with antifactor Xa assays.

\section{REFERENCES}

1. Gould MK, Garcia DA, Wren SM, et al. Prevention of VTE in nonorthopedic surgical patients: antithrombotic therapy and prevention of thrombosis, 9th ed: American College of Chest Physicians Evidence-Based Clinical Practice guidelines. Chest 2012;141:e227S-77S. [PubMed: 22315263]

2. Malinoski D, Jafari F, Ewing T, et al. Standard prophylactic enoxaparin dosing leads to inadequate anti-Xa levels and increased deep venous thrombosis rates in critically ill trauma and surgical patients. J Trauma 2010;68:874-80. [PubMed: 20386282]

3. Nunez JM, Becher RD, Rebo GJ, et al. Prospective evaluation of weight-based prophylactic enoxaparin dosing in critically ill trauma patients: adequacy of AntiXa levels is improved. Am Surg 2015;81:605-9. [PubMed: 26031274] 
4. Geerts WH, Pineo GF, Heit JA, et al. Prevention of venous thromboembolism: the Seventh ACCP Conference on Antithrombotic and Thrombolytic therapy. Chest 2004;126:338S-400S. [PubMed: 15383478]

5. White AE, Edelman JB, Lott N, et al. Characterization of the hypercoagulable state following severe orthopedic trauma. J Trauma Acute Care Surg 2014;77:231-7. [PubMed: 25058247]

6. Geerts WH, Code KI, Jay RM, et al. A prospective study of venous thromboembolism after major trauma. N Engl J Med 1994; 331:1601-6. [PubMed: 7969340]

7. Paffrath T, Wafaisade A, Lefering R, et al. Venous thromboembolism after severe trauma: incidence, risk factors and outcome. Injury 2010;41:97-101. [PubMed: 19608183]

8. Toker S, Hak DJ, Morgan SJ. Deep vein thrombosis prophylaxis in trauma patients. Thrombosis 2011;2011:505373. [PubMed: 22084663]

9. Geerts WH, Jay RM, Code KI, et al. A comparison of low-dose heparin with low-molecular-weight heparin as prophylaxis against venous thromboembolism after major trauma. N Engl J Med 1996;335:701-7. [PubMed: 8703169]

10. National Trauma Databank. Available at: https://www.ntdbdatacenter.com/MemberLogin.aspx.

11. Demuro JP, Hanna AF. Prophylaxis of deep venous thrombosis in trauma patients: a review. J Blood Disorders Transf 2013; 4:1000151.

12. Iqbal Z, Cohen M. Enoxaparin: a pharmacologic and clinical review. Expert Opin Pharmacother 2011;12:1157-70. [PubMed: 21470072]

13. Samama MM, Poller L. Contemporary laboratory monitoring of low molecular weight heparins. Clin Lab Med 1995;15: 119-23. [PubMed: 7781274]

14. Duplaga BA, Rivers CW, Nutescu E. Dosing and monitoring of low-molecular-weight heparins in special populations. Pharmacotherapy 2001;21:218-34. [PubMed: 11213859]

15. Costantini TW, Min E, Box K, et al. Dose adjusting enoxaparin is necessary to achieve adequate venous thromboembolism prophylaxis in trauma patients. J Trauma Acute Care Surg 2013; 74:128-33. [PubMed: 23271087]

16. ACS Committee on Trauma. ACS NTDB National Trauma Data Standard: Data Dictionary for 2016 admissions. Available at: http://www.ntdsdictionary.org/softwareVendors/documents/ NTDSDataDictionary-2016Admissions_11042015.pdf.

17. Rossaint R, Bouillon B, Cerny V, et al. The European guideline on management of major bleeding and coagulopathy following trauma: fourth edition. Crit Care 2016;20:100. [PubMed: 27072503]

18. Brohi K, Cohen MJ, Davenport RA. Acute coagulopathy of trauma: mechanism, identification, and effect. Curr Opin Crit Care 2007;13:680-85. [PubMed: 17975390]

19. Rizoli SB, Scarpelini S, Callum J, et al. Clotting factor deficiency in early trauma-associated coagulopathy. J Trauma 2011;71:S427-34. [PubMed: 22071999]

20. Gonzalez E, Moore EE, Moore HB, et al. Trauma-Induced coagulopathy: an institution's 35 year perspective on practice and research. Scand J Surg 2014;103:89-103. [PubMed: 24786172]

21. Simmons JW, Pittet JF, Pierce B. Trauma-Induced coagulopathy. Curr Anesthesiol Rep 2014;4:189-99. [PubMed: 25587242]

22. D'Angelo MR, Dutton RP. Management of trauma-induced coagulopathy: trends and practices. AANA J 2010;78: 35-40. [PubMed: 20977127]

23. Robinson S, Zincuk A, Larsen UL, et al. A comparative study of varying doses of enoxaparin for thromboprophylaxis in critically ill patients: a double-blinded, randomised controlled trial. Crit Care 2013;17:R75. [PubMed: 23601744]

24. Mayr AJ, Dünser M, Jochberger S, et al. Antifactor Xa activity in intensive care patients receiving thromboembolic prophylaxis with standard doses of enoxaparin. Thromb Res 2002;105:201-4. [PubMed: 11927124] 


\section{Trauma Services VTE Prophylaxis Algorithm}

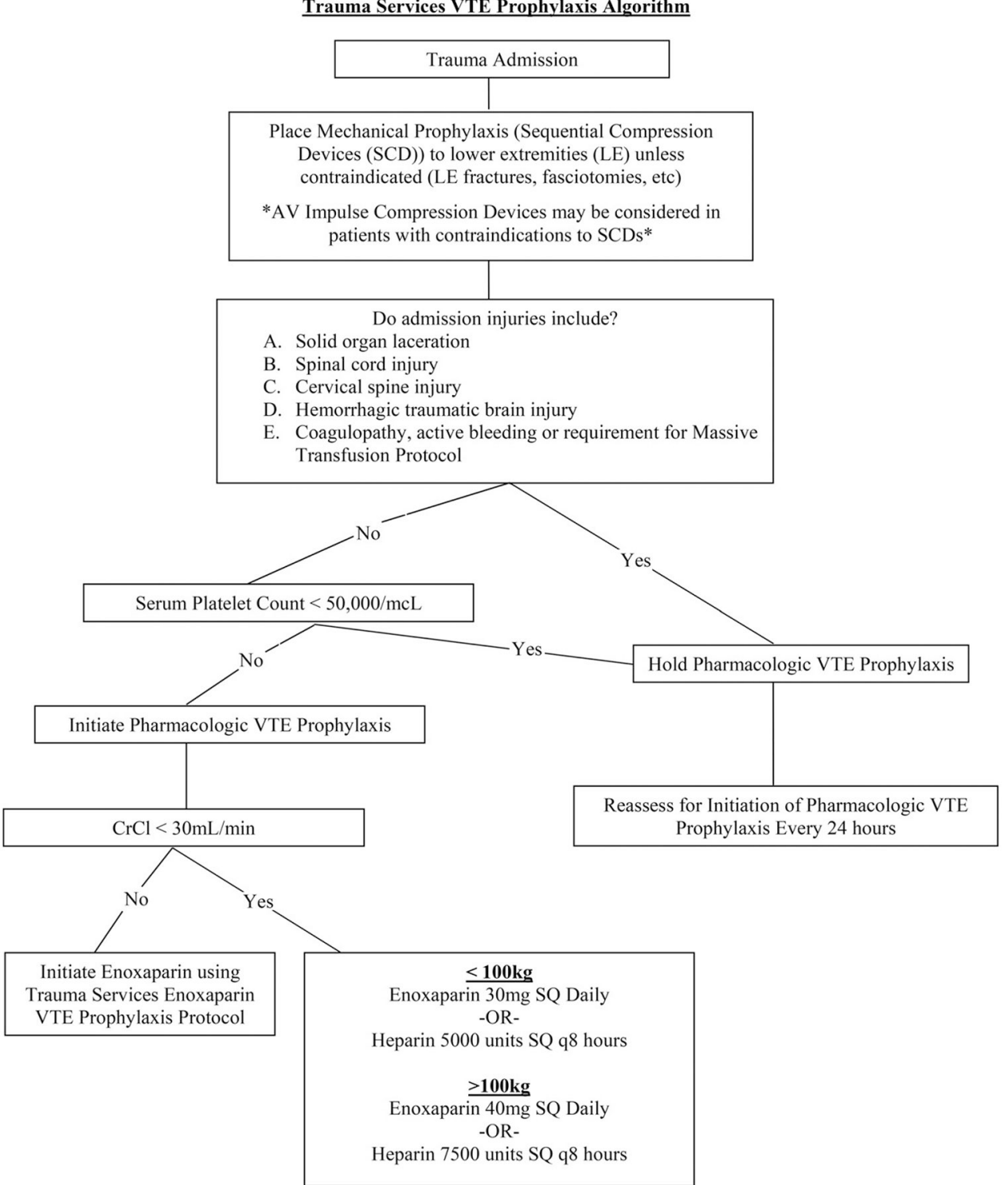

Fig. 1.

Trauma Services VTE Prophylaxis Algorithm. 


\section{$\underline{\text { Trauma Services Enoxaparin VTE Prophylaxis Protocol }}$}

1. Enoxaparin dosing scheduled at $12: 00$ and 00:00

- $\quad<100 \mathrm{~kg} \rightarrow 30 \mathrm{mg}$ SQ BID $\quad$ or $\quad>100 \mathrm{~kg} \rightarrow 40 \mathrm{mg}$ SQ BID

2. Check an anti-factor Xa level (Lovenox Heparin Assay) after $3^{\text {rd }}$ or $4^{\text {th }}$ dose (with a.m. labs)

- If sub-therapeutic $(<0.2 \mathrm{IU} / \mathrm{mL}) \rightarrow$ Increase each dose by $20 \%$ (each dose rounded to the nearest zero)

- If super-therapeutic $(>0.5 \mathrm{IU} / \mathrm{mL}) \rightarrow$ Decrease each dose by $20 \%$ (each dose rounded to the nearest zero)

$>$ Regimens of $30 \mathrm{mg}$ SQ q12h should be changed to once daily regimens $-\quad<100 \mathrm{~kg} \rightarrow 30 \mathrm{mg}$ SQ qday $\quad$ or $\quad>100 \mathrm{~kg} \rightarrow 40 \mathrm{mg}$ SQ qday

3. After each dosing change (BID regimens only), repeat anti-factor Xa level (Lovenox Heparin Assay) after the $3^{\text {rd }}$ or $4^{\text {th }}$ dose and adjust accordingly as outlined above

4. Protocol management is contraindicated for any of the following reasons:

- Renal insufficiency (GFR/CrCl $<30 \mathrm{~mL} / \mathrm{min}$ ), thrombocytopenia $<50,000 / \mathrm{mcL}$, injuries/clinical scenarios as outlined in "Trauma Service's VTE Algorithm"

Fig. 2.

Trauma Services Enoxaparin VTE Prophylaxis Protocol. 




Am Surg. Author manuscript; available in PMC 2019 June 01. 
BETHEA et al.

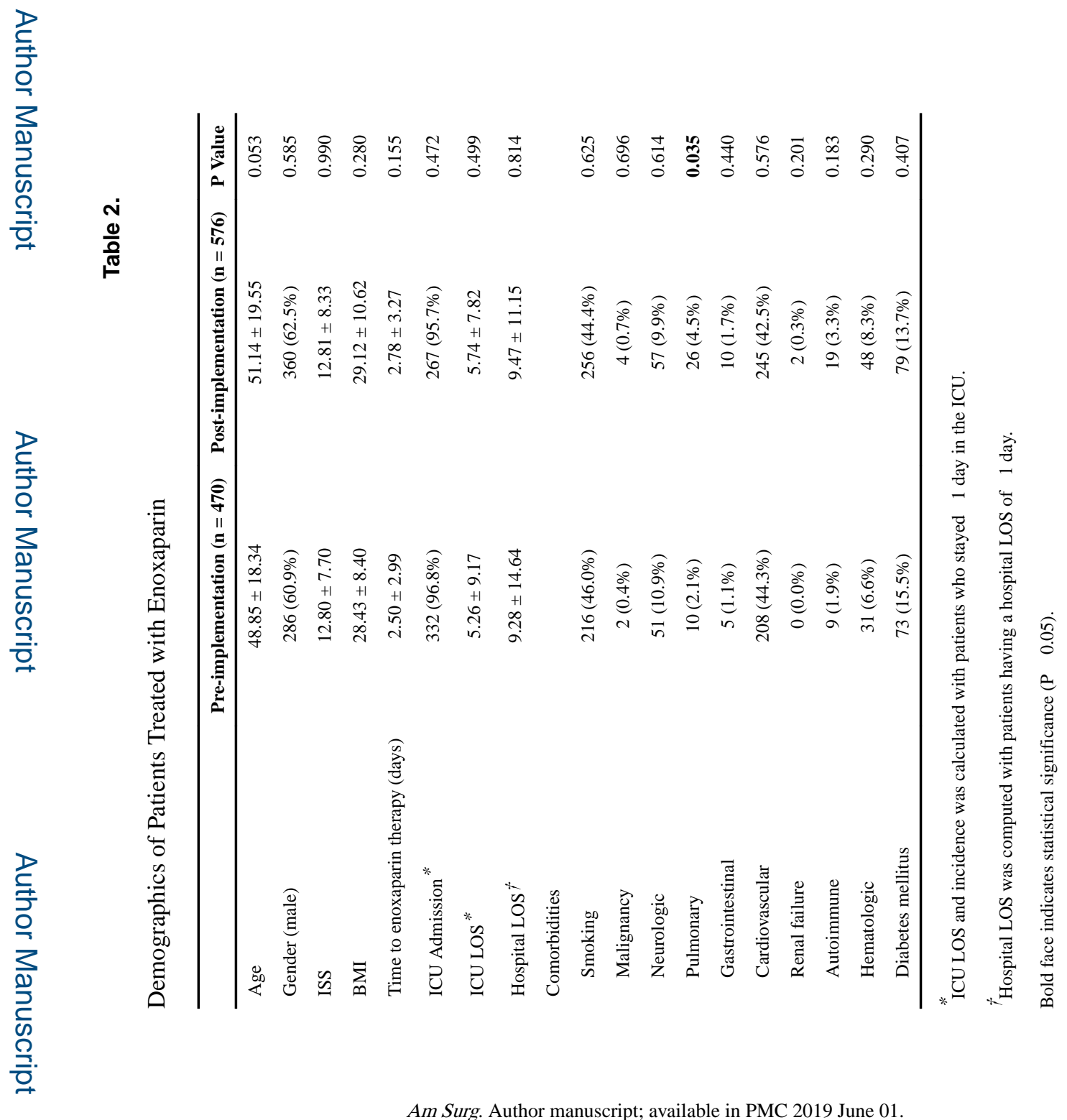




\section{롤 \\ 골}

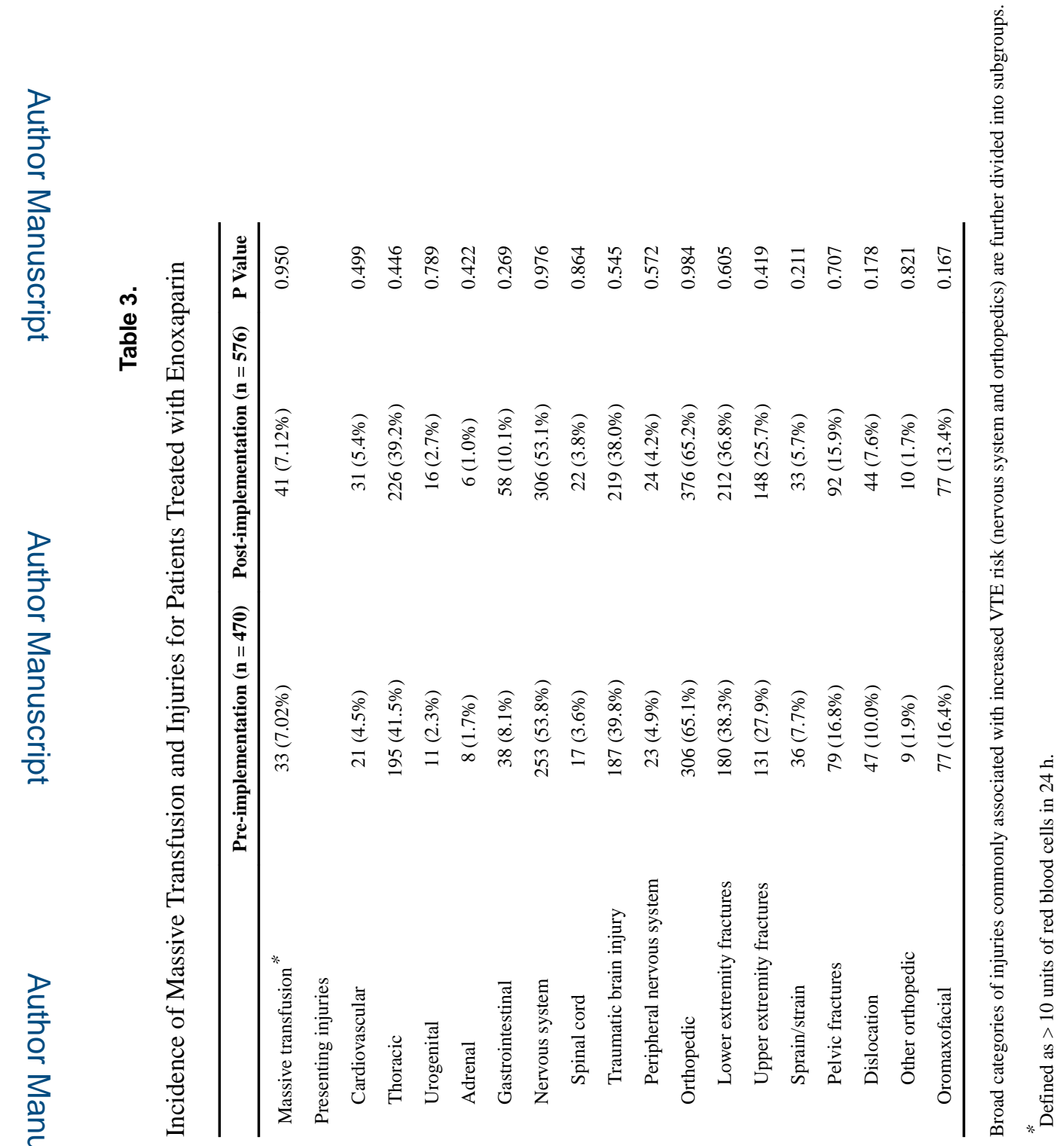

Am Surg. Author manuscript; available in PMC 2019 June 01. 


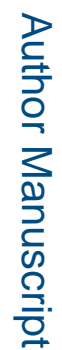

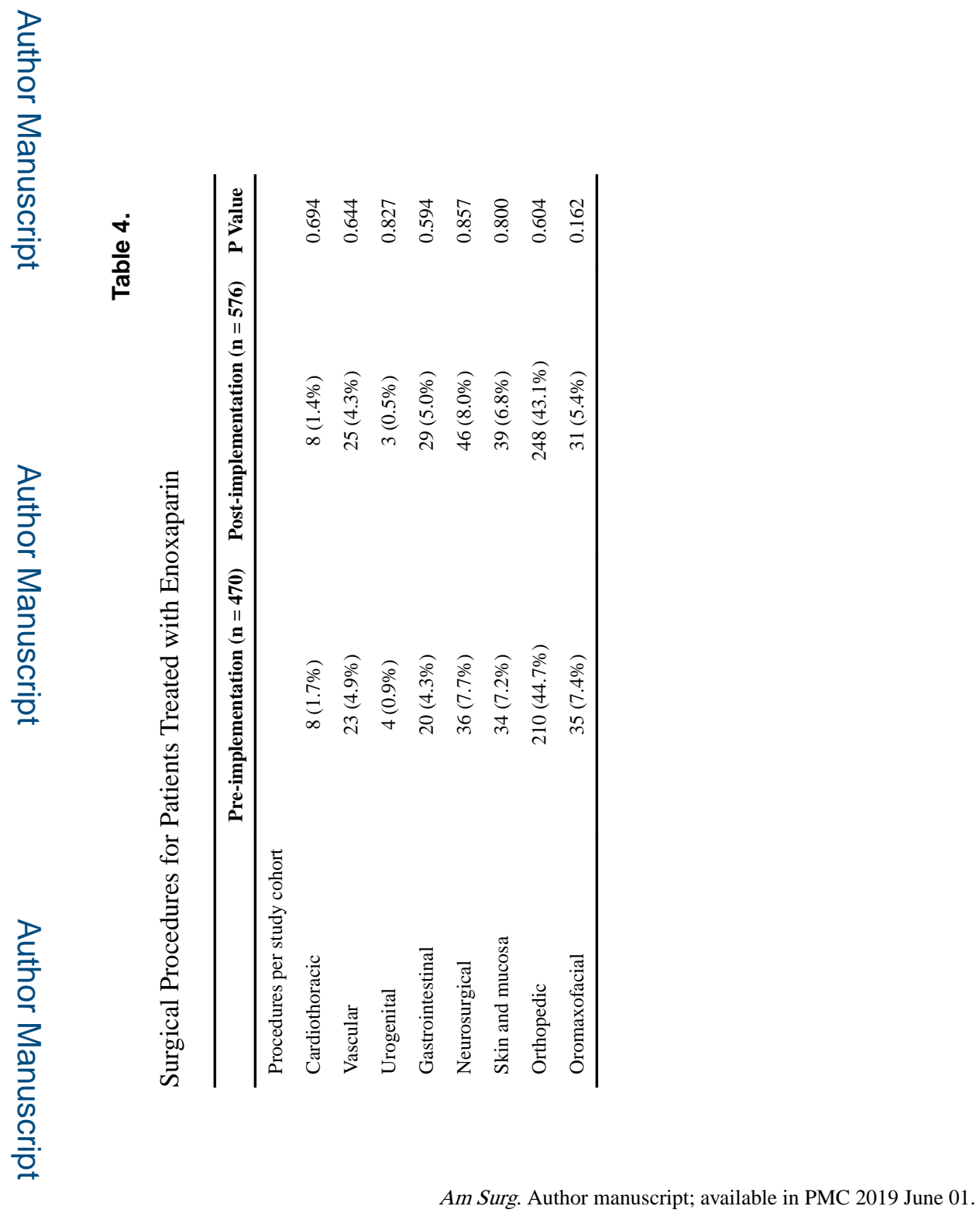



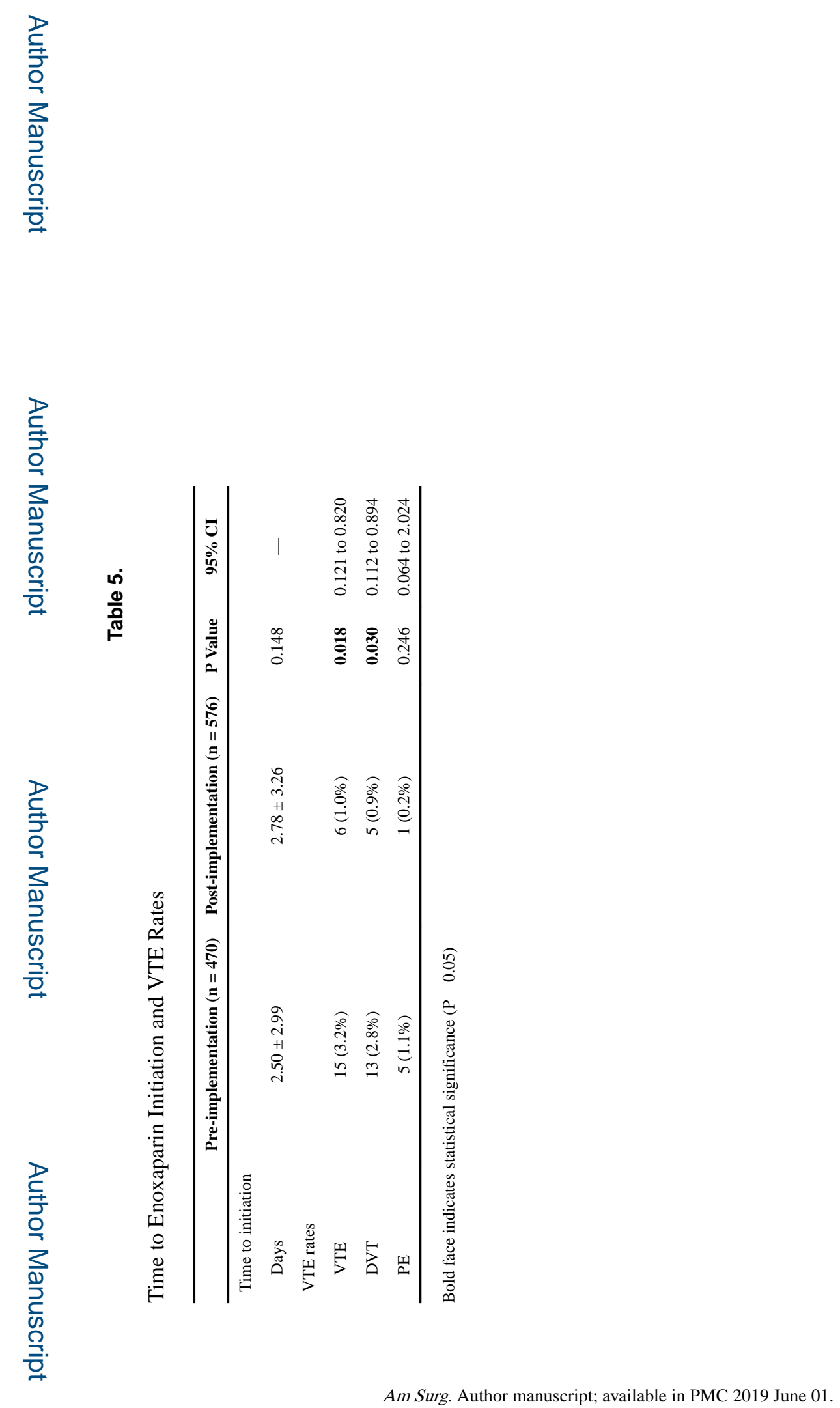


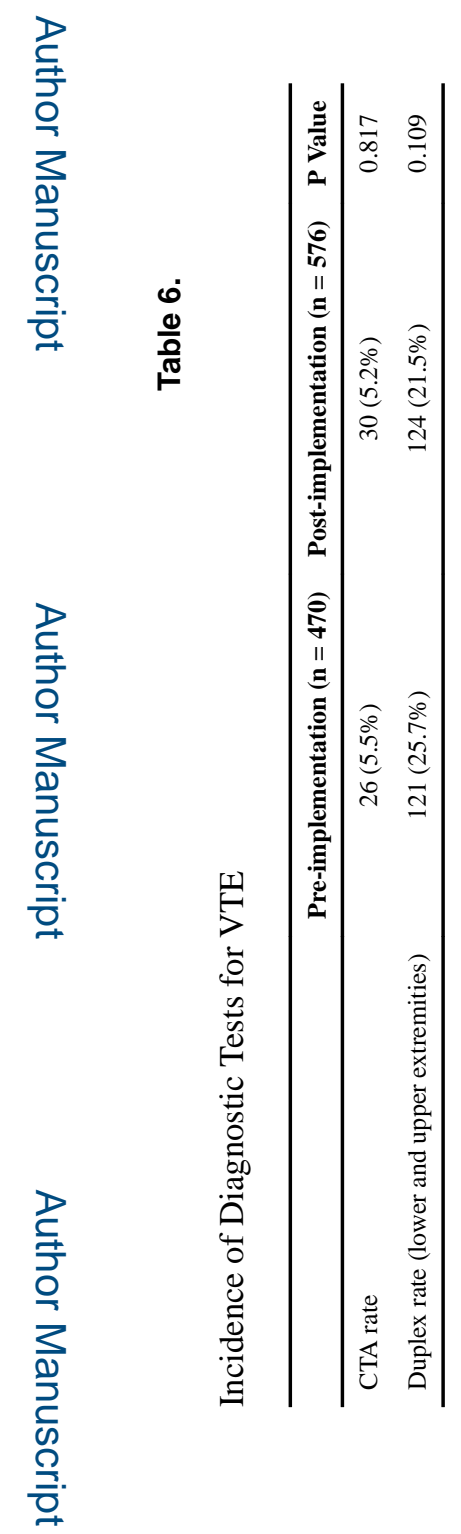

Am Surg. Author manuscript; available in PMC 2019 June 01. 